\title{
Cerebrospinal fluid Neurofilament Light is associated with survival in mitochondrial disease patients
}

Kalliopi Sofou $^{\dagger, a}$, Pashtun Shahim ${ }^{\dagger,}$,c, , Már Tuliniuss $^{\mathrm{a}}$, Kaj Blennow ${ }^{\mathrm{b}, \mathrm{c}}$, Henrik Zetterberg $^{\mathrm{b}, \mathrm{c}, \mathrm{d}, \mathrm{e}}$, Niklas Mattsson ${ }^{\mathrm{f}}$, Niklas Darin ${ }^{\mathrm{a}}$

aDepartment of Pediatrics, University of Gothenburg, The Queen Silvia's Children Hospital, Gothenburg, Sweden

${ }^{b}$ Institute of Neuroscience and Physiology, Department of Psychiatry and Neurochemistry, the Sahlgrenska Academy at University of Gothenburg, Mölndal, Sweden.

${ }^{c}$ Clinical Neurochemistry Laboratory, Sahlgrenska University Hospital, Mölndal, Sweden

${ }^{\mathrm{d}}$ Department of Molecular Neuroscience, UCL Institute of Neurology, Queen Square, London, UK

${ }^{\mathrm{e}}$ UK Dementia Research Institute at UCL, London, UK

${ }^{f}$ Clinical Memory Research Unit, Lund University, Malmö, Sweden, and Lund University, Skåne University Hospital, Department of Clinical Sciences, Neurology, Lund, Sweden

${ }^{\dagger}$ Equal contributors

Correspondence to: Kalliopi Sofou, $\mathrm{MD}, \mathrm{PhD}$

Department of Pediatrics

University of Gothenburg,

The Queen Silvia's Children Hospital

SE-416 85 Gothenburg, Sweden

Tel: +46 (0) 313421000

E-mail: kalliopi.sofou@vgregion.se

\section{Abbreviations}

\begin{tabular}{|l|l|}
\hline Ab42 & Amyloid-b42 \\
\hline AD & Alzheimer's disease \\
\hline AUROC & Area under the receiver operating-characteristic curve \\
\hline CNS & Central nervous system \\
\hline CSF & Cerebrospinal fluid \\
\hline DWI & Diffusion weighted imaging \\
\hline FLAIR & Fluid attenuated inversion recovery \\
\hline GFAp & Glial fibrillary acidic protein \\
\hline KSS & Kearns-Sayre syndrome \\
\hline LP & Lumbar puncture \\
\hline ME & Mitochondrial encephalopathy \\
\hline MELAS & $\begin{array}{l}\text { Mitochondrial encephalomyopathy, lactic acidosis and } \\
\text { stroke-like episodes }\end{array}$ \\
\hline MERRF & Myoclonic epilepsy with ragged red fibers \\
\hline
\end{tabular}




\begin{tabular}{|l|l|}
\hline MRI & Magnetic resonance imaging \\
\hline MRS & Magnetic resonance spectroscopy \\
\hline NARP & Neuropathy, ataxia, and retinitis pigmentosa \\
\hline NF-L & Neurofilament light \\
\hline PDC & Pyruvate dehydrogenase complex \\
\hline P-tau & Phosphorylated tau \\
\hline TBI & Traumatic brain injury \\
\hline T-tau & Total tau \\
\hline
\end{tabular}




\begin{abstract}
We studied the biomarker patterns related to axonal injury, astrogliosis and amyloid metabolism in cerebrospinal fluid (CSF) of children and adolescents with mitochondrial encephalopathy and identified correlations with phenotype and survival outcome. Forty-six pediatric patients with genetically verified mitochondrial encephalopathy and twenty-two controls investigated at the Queen Silvia Children's Hospital, Sweden, were included. CSF lactate and neurofilament light (NF-L) were significantly increased in patients with mitochondrial encephalopathy compared to controls. Elevated CSF NF-L was associated with abnormal brain MRI and poorer survival. We suggest that CSF NF-L may be used in both clinical and research settings for monitoring the neurodegenerative process in mitochondrial disease.
\end{abstract}

Keywords: Mitochondrial disease; biomarker; neurofilament; cerebrospinal; neuroimaging; neurodegenerative 


\section{Introduction}

Mitochondrial diseases are a group of clinically and genetically heterogeneous disorders caused by dysfunction in the mitochondria, leading to impaired cellular energy production [Moslemi and Darin, 2007]. Mitochondrial disease can present at any age with symptoms from any organ or tissue although the central nervous system (CNS) and the skeletal muscles are typically more severely affected owing to their high-energy demands. In infancy and early childhood the disease is often multi-systemic, characterized by progressive encephalopathy, refractory seizures, psychomotor regression, failure to thrive, muscular hypotonia, movement disorder, cardiomyopathy or liver failure [Tulinius et al., 1991a].

The underlying genetics of mitochondrial diseases is either mutations in the mitochondrial DNA or nuclear DNA [Moslemi and Darin, 2007; Tulinius et al., 1991b]. The range of mitochondrial and nuclear DNA mutations that impair the assembly, synthesis or maintenance of proteins necessary for the function of the mitochondrial respiratory chain is large and growing. Mitochondrial dysfunction due to mechanisms that are not yet completely understood may also play an important role in numerous degenerative diseases, including amyotrophic lateral sclerosis and Alzheimer's disease (AD) [Lin and Beal, 2006; Reddy, 2009].

Due to the multifaceted and complex nature of mitochondrial diseases, the diagnostic process, especially in the pediatric population, can be highly challenging. At present, the diagnostic arsenal includes neuroimaging upon suspicion of CNS involvement, analysis of blood, urine and cerebrospinal fluid (CSF), muscle biopsy, skin biopsy, and molecular genetics [Haas et al., 2008]. Neuroimaging typically includes structural magnetic resonance imaging (MRI) of the brain, diffusion weighted imaging (DWI) and magnetic resonance spectroscopy (MRS). Combined with clinical indices, neuroimaging has great diagnostic utility in mitochondrial 
diseases. However, especially in early stages of disease, neuroimaging can be normal or show non-specific abnormalities [Sofou et al., 2013]. The muscle biopsy may show normal respiratory chain enzyme activities as respiratory chain defects are not always expressed in the skeletal muscle, or non-specific histopathological findings [Sofou et al., 2014].

Fluid biomarkers such as lactate levels in blood and CSF are important but non-specific markers of mitochondrial disease. Many patients with mitochondrial disease may have normal lactate levels unless undergoing metabolic crisis or following exercise [Sofou et al., 2014; Debray et al., 2007]. Other CSF biomarkers have been extensively studied in neurological diseases. For example, CSF biomarkers reflecting amyloid and tau pathology have been studied in the context of $\mathrm{AD}$, and are now used in clinical practice to support diagnosis and prognosis of AD [Olsson et al., 2016]. Recently, studies have also assessed the axonal injury biomarker neurofilament light protein (NF-L) in neurodegenerative diseases, including AD, as well as individuals with traumatic brain injury (TBI), where CSF NF-L shows both diagnostic and prognostic utility [Zetterberg et al., 2016; Skillback et al., 2014; Shahim et al., 2016a; Shahim et al., 2016b; Zetterberg et al., 2006].

As mitochondrial diseases with CNS involvement often present as a progressive neurodegenerative disease, we assessed whether CSF biomarkers of axonal and astroglial injury, as well as amyloid metabolism would also be altered in children and adolescents with mitochondrial diseases [Lin and Beal, 2006; Reddy, 2009]. We specifically tested the hypotheses that CSF biomarkers of axonal and astroglial injury, and amyloid metabolism, would (1) have a greater clinical utility for mitochondrial disease compared with the conventionally used CSF lactate, and (2) correlate with overall survival. We expected the effects to be strongest for CSF NF-L, because of the promising findings for this biomarker in 
other conditions.

\section{Methods}

\subsection{Ethical Approval}

The study was approved by the Regional Ethics Committee at the University of Gothenburg, Sweden. Signed informed consent was obtained for the publication of MRI images by the patient's guardian.

\subsection{Study Population}

This was a cross-sectional study, involving children and adolescents $(\mathrm{N}=594)$ referred to the Queen Silvia Children's Hospital during the period 1984 - 2014 for suspected mitochondrial disease. As part of the standard medical assessment upon referral, all patients underwent clinical investigation, muscle biopsy and laboratory investigations, while neuroimaging was performed in patients with CNS disease when applicable. All patients underwent medical and family history assessment for other hereditary disorders. Patients diagnosed with mitochondrial disease underwent annual clinical follow-ups. In our study, we included follow-up data until the end of December 2016.

The patient flow is shown in Figure 1. Sixteen patients without mitochondrial respiratory chain deficiencies or CNS disease were included as controls [Darin et al., 2001].

\subsection{Brain Imaging}

A total of 35 patients were radiologically assessed based on a specific MRI protocol, including conventional T1- and T2-weighted images. Axial and coronal T2-weighted images, including fluid attenuated inversion recovery (FLAIR) images, were assessed to identify changes in signal intensity. Additional imaging sequences, such as DWI and MRS, were performed when applicable to help differentiate among observed signal changes and detect metabolic 
abnormalities. Abnormal MRI findings were categorized as abnormal signal intensity, atrophy, hemorrhage, calcifications, abnormal ventricular size, dysgenesis/agenesis of the corpus callosum and abnormal myelination. The findings were topographically categorized as focal/diffuse/global and unilateral/bilateral. In the case of focal findings, these were categorized on the basis of their predominant localization, i.e. cerebral cortex; supratentorial white matter; corpus callosum, basal ganglia, diencephalon, midbrain, pons, medulla oblongata and cerebellum.

\subsection{Biochemical, Morphological and Genetic Analyses}

All patients underwent muscle biopsy obtained from the vastus lateralis muscle as described elsewhere [Lin and Beal, 2006]. Polarographic and spectrophotometric measurements of the respiratory chain were performed in isolated mitochondria, while morphological, histochemical, immunohistochemical and electron microscopy analyses were performed on fresh-frozen muscle tissue. A skin biopsy was performed at the same time to obtain fibroblasts for molecular analyses. Genetic investigations included a panel of common mitochondrial DNA point mutations (mitochondrial encephalomyopathy, lactic acidosis and stroke-like episodes [MELAS], myoclonic epilepsy with ragged red fibers [MERRF], neuropathy, ataxia, and retinitis pigmentosa $[\mathrm{NARP}])$, and if negative, sequencing of the entire mitochondrial genome was performed. Once a causative mutation in mitochondrial DNA was excluded, whole exome sequencing was performed.

\subsection{CSF Biomarker Analysis}

CSF was collected in polypropylene tubes by lumbar puncture (LP) through the L3-4 or L4-5 interspace. All CSF samples were stored at $-80^{\circ} \mathrm{C}$ pending analysis. The xMAP assay INNOTEST AlzBio3 (Fujirebio, Ghent, Belgium) was used for quantification of A $\beta 1-42$, T-tau, and 
P-tau, as described previously [Olsson et al., 2005]. NFL was analyzed using NF-light ELISA from UmanDiagnostics (Umeå, Sweden). GFAp concentration was measured using a previously described in house ELISA [Rosengren et al., 1994]. Lactate concentrations in blood and CSF were measured using enzyme-based spectrophotometric assay. All samples were analyzed at the same time using the same batch of reagents by board-certified laboratory technicians who were blinded to clinical information.

\subsection{Statistical Analysis}

The Mann-Whitney $U$ test and $\chi^{2}$ test was used to examine differences in continuous variables and categorical variables between the mitochondrial disease groups versus healthy controls. Linear regression models were constructed for comparison between the subgroups of mitochondrial disease and controls co-varied for age at the time of LP and sex. The subgroup analysis was based on distinct phenotypic subgroups summarized in Supplementary Table 1. Comparison of biomarker concentrations between the various genetic subgroups was not feasible due to the limited number of patients per genetic subgroup. For association between the MRI findings and biomarkers concentrations, linear regression models were constructed co-varied for age at the time of LP and sex. We assessed the overall diagnostic utility of the biomarkers by assessing the area under the receiver operating-characteristic curve (AUROC). Survival analysis was conducted using Cox regression, where the last known date alive was used for censoring when date of death was unknown, and biomarker, age, sex, and diagnosis, were used as predictors. All tests were two-sided and statistical significance was determined at $P<0.05$ (two-sided). All statistical analyses were performed using $\mathrm{R}$ (v. 3.0.3, The $\mathrm{R}$ Foundation for Statistical Computing).

\section{Results}

\subsection{Demographic Characteristics}


A total of 52 patients (23 males; 29 females) with clinically and genetically verified mitochondrial disease, 46 with mitochondrial encephalopathy and 6 with mitochondrial myopathy without CNS involvement, were included in the final study cohort along with 16 controls (10 males; 6 females) (Figure 1). There was no significant difference in the levels of CSF biomarkers between patients with mitochondrial myopathy $(n=6)$ and controls $(n=16$; data not shown). We therefore included the mitochondrial myopathy patients in the control group $(n=22)$. The phenotype and genetic defects of patients with mitochondrial encephalopathy (ME, n=46) are summarized in Table 2. An overview of the syndromic and non-syndromic mitochondrial encephalopathies is provided in Supplementary Table 1.

\subsection{Increased CSF NF-L in Mitochondrial Encephalopathy}

The levels of CSF lactate were mildly increased in patients with ME compared with controls $(P<0.001$, Figure 2 and Table 1). However, there was no difference in the levels of blood lactate between the groups $(P=0.21$; Figure 2 and Table 1). CSF lactate could differentiate between patients with ME and controls with AUROC 0.88, while blood lactate had AUROC 0.60 (Figure 3). The CSF levels of NF-L showed a marked increase in the ME group compared with controls $(P<0.001$; Figure 2 and Table 1). CSF NF-L could separate between patients with ME and controls with AUROC 0.90 (Figure 3). There were no significant differences in the concentrations of the other biomarkers between patients with ME and controls (Figure 3 and Table 1).

In a subgroup analysis, significantly increased CSF lactate was observed in all the subgroups of ME patients except MERRF and non-syndromic ME (Table 2). Increased concentrations of NF-L were found in patients with Leigh syndrome, MELAS, and pyruvate dehydrogenase complex (PDC) deficiency as compared to controls (Table 2). Patients with PDC deficiency 
and Kearns-Sayre syndrome (KSS) had reduced concentrations of A $\beta 42$ compared with controls, with the latter also having increased concentrations of P-tau as well (Table 2). There were no significant differences in the CSF concentrations of T-tau or GFAp (Table 2).

\subsection{Associations Between Abnormal CSF and MRI Biomarker Findings and Diagnosis}

Of the 35 assessed patients, abnormal MRI findings were found in 28 patients upon referral. These included either one of the following regions: cerebral cortex $(n=12)$, basal ganglia $(n=12)$, diencephalon $(n=12)$, supratentorial white matter $(n=10)$, cerebellum $(n=8)$, midbrain $(n=7)$, medulla oblongata $(n=5)$, pons $(n=4)$ and corpus callosum $(n=4)$. The majority of the MRI investigations showed increased signal intensity on T2-weighted images in singular or combined regions of the brain $(n=20$, Figure 4$)$, while atrophy without signal changes was found in 5 patients, all with PDC deficiency. An overview of the brain regions affected in each mitochondrial subgroup is shown in Table 2. Overall, the patients with abnormal brain MRI had increased concentrations of CSF NF-L as compared to those with normal MRI $(P=0.001)$. Specifically, increased NF-L was associated with abnormalities in the cerebral cortex $(P=$ 0.027), medulla $(P=0.014)$, and midbrain $(P=0.0003)$. The overall concentrations of the other biomarkers did not differ significantly between patients with normal and abnormal MRI. However, increased CSF lactate concentrations showed associations with MRI abnormalities in cortex $(P=0.0016)$, while abnormalities in medulla and midbrain were also associated with increased concentrations of CSF T-tau and GFAp $(P<0.001$, all $)$. There was no significant association between the MRI findings and concentrations of P-tau and A $\beta 42$.

\subsection{NF-L was Predictive of Outcome in Patients with Mitochondrial Encephalopathy}

There was no significant difference in the concentrations of the biomarkers between the survivor and non-survivors, except for CSF NF-L $(P<0.001)$. There was no association 
between CSF lactate concentrations and poorer survival with estimated hazard ratio (HR) of 1.4 (95 \% CI 0.8-2.5, $P=0.25$; Figure 5A). In contrast, higher CSF NF-L levels were associated with poorer survival adjusted for age, diagnosis, and sex, with estimated HR of 3.23 (95\% CI 1.5-7.0, $P=0.0030$; Figure 5B). Higher concentrations CSF GFAp were associated with poorer survival with estimated HR of 2.7 (95\% CI 1.25-5.8, $P=0.011$; Figure 5C-D). There was no significant association between the CSF concentrations of P-tau or A $\beta 42$ and survival (Supplementary figure 1). 


\section{Discussion}

In this study, we present the CSF biomarker profile in relation to underlying phenotypic, genotypic and imaging findings in a large cohort of children and adolescents with mitochondrial disease. We found that: 1) CSF lactate was increased in patients with ME as compared with controls, however, the increase was mild to moderate, and did not show any association to outcome, 2) CSF NF-L was significantly increased in patients with ME and was associated with poorer survival outcome; (3) specifically patients with Leigh syndrome, MELAS and PDC deficiency had a marked increase in the concentrations of CSF NF-L as compared to controls, 4) CSF GFAp did not differ between patients with ME and controls, but was associated with poorer survival outcome, and 5) CSF NF-L was the only biomarker associated with abnormal MRI findings in patients with ME.

Elevated CSF lactate has been proposed as a reliable diagnostic biomarker for mitochondrial diseases [Magner et al., 2011]. In the present study, we found increased CSF lactate in patients with ME as compared to controls, without any association to outcome. Our results are in agreement with the results from another recent study, suggesting that CSF lactate is a less reliable biomarker for mitochondrial diseases [Lunsing et al., 2017].

NF-L is a structural protein that is highly expressed in the large-caliber myelinated subcortical axons of the white matter [Zetterberg et al., 2016; Skillback et al., 2014]. Our finding that CSF NF-L increases in patients with mitochondrial disease is in concordance with previous studies of CSF NF-L in axonal degenerative disease such as AD, multiple sclerosis, amyotrophic lateral sclerosis, and frontotemporal dementia [Zetterberg et al., 2016; Skillback et al., 2014; Magnoni et al., 2012; Tortorella et al., 2015; Lu et al., 2015]. Also, increased CSF NF-L has been reported in patients with ischemic stroke, as well as traumatic brain injury [Shahim et al., 
2016b; Nylen et al., 2006]. Among patients with mitochondrial disease, significantly increased concentrations of CSF NF-L were observed in patients with Leigh syndrome, MELAS and PDC deficiency. Patients with PDC deficiency often exhibit severe decrease in white matter volume, and in turn dilated ventricles with ventricular septations, or parenchymal cysts and partial to complete agenesis of the corpus callosum [Sofou et al., 2013; Ah Mew et al., 2011]. An ongoing white matter degeneration may explain the increased NF-L levels seen in these patients. A plausible explanation to the increase in CSF NF-L in Leigh syndrome and MELAS could be that these diseases are characterized by cytotoxic edema in the cortical (MELAS) or deep gray matter (Leigh syndrome) in either the acute or subacute stages of the disease [Sofou et al., 2013; Cecchini et al., 2007]. In contrast to lactate that may normalize after an increase following metabolic decompensation, it seems from other studies of neurodegenerative diseases that release of CSF NF-L is associated with the progression of neurodegeneration [Zetterberg et al., 2016; Mattsson et al., 2017].

We found CSF NF-L to be significantly increased in patients with abnormal brain MRI as compared to those with normal MRI, more specifically in relation to abnormalities in the cerebral cortex, medulla and midbrain. Interestingly, our patient with Alpers-Huttenlocher syndrome had normal brain MRI but higher levels than controls in all biomarkers, especially NF-L, but normal lactate. Our findings suggest that NF-L may serve as an early prognostic marker, much better than lactate, indicating signs of axonal injury in the brain already in the early phase of the disease and before these become apparent on neuroimaging.

Another CSF protein measured was GFAp which is predominantly expressed in astrocytes and plays a role in the formation of astroglial scaring [Shahim et al., 2013a]. Overall there were no significant changes in the level of CSF GFAp between patients with ME and controls. 
However, in the subgroup analysis, patients with KSS had significantly increased concentrations of CSF GFAp. Also, increased CSF GFAp was associated with poorer survival. A previous study has reported increased CSF GFAp in patients with Alexander disease, which is characterized by white matter degeneration or astrogliosis [Shahim et al., 2013a]. Similar to Alexander disease, patients with KSS may also display white matter cerebral abnormalities, which could explain the increased concentrations of CSF GFAp in these cases [Serrano et al., 2010].

Similar to lactate, we did not find any changes in the concentrations of T-tau or P-tau. A plausible explanation could be that mitochondrial disease may foremost affect the white matter and deeper brain structures rather than the superficial cortex where tau is predominantly expressed. The lack of effect on $\mathrm{A} \beta 42$ argues against significant abnormalities in or $\mathrm{A} \beta$ metabolism in these patients. Similar results, where the concentrations of NF-L, but not T-tau or P-tau or A $\beta 42$ have been elevated in adults with TBI [Shahim et al., 2016b; Zetterberg et al., 2006].

There are limitations to this study. Although the overall sample size is large considering the rarity of mitochondrial diseases, our results in the subgroup analysis should be interpreted with caution due to the small number of patients per subgroup. Furthermore, comparison at the single disease level could not be performed for specific phenotypes such as AlpersHuttenlocher and leukoencephalopathy. Additional limitations include lack of follow-up CSF sampling on all the patients allowing biochemically monitoring of the progression of the disease. 
The results of this study suggest that CSF NF-L may be used in the clinical setting as a biomarker of diagnostic and prognostic value in ME. Additionally, CSF NF-L may also be used in clinical trials for selection and evaluation of novel therapies for mitochondrial diseases. 


\section{Acknowledgement}

We warmly acknowledge the patients and their families. We also would like to thank Lobna Almasalmeh, BSc for her technical assistance with the analysis of the CSF samples.

\section{Author contribution}

Drs Shahim and Sofou had full access to all the data in the study and take responsibility for the integrity of the data and the accuracy of the data analysis.

Study concept and design: Sofou, Tulinius, Zetterberg, Darin.

Acquisition of data: Shahim, Sofou, Tulinius, Blennow, Zetterberg, Darin.

Statistical analysis: Shahim, Sofou and Mattsson.

Drafting of the manuscript: Shahim, Sofou, Mattsson.

Analysis and interpretation of data: Shahim, Sofou, Tulinius, Blennow, Zetterberg, Mattsson, Darin.

Critical Revision of the manuscript for important intellectual content: All authors.

Administrative, technical or material support: Blennow, Zetterberg.

Obtained funding: Blennow, Zetterberg, Darin.

Study Supervision: Zetterberg, Mattsson, Darin.

\section{Study funding}

The study was supported by grants from the Swedish Research Council, the European Research Council, the Torsten Söderberg Foundation, the Knut and Alice Wallenberg Foundation, Frimurarestiftelsen and AnnMari and Per Ahlqvist Foundation.

The funding source had no role in the design and conduct of the study; collection, management, analysis, or interpretation of the data; and preparation, review, or approval of the manuscript; and decision to submit the manuscript for publication. 


\section{Disclosure}

H. Zetterberg is a co-founder of Brain Biomarker Solutions in Gothenburg AB, a GU Venturesbased platform company at the University of Gothenburg and has served at advisory boards for Roche Diagnostics and Eli Lilly. K. Blennow has served as a consultant or at advisory boards for Alzheon, Eli Lilly, Fujirebio Europe, IBL International, Pfizer, and Roche Diagnostics, and is a co-founder of Brain Biomarker Solutions in Gothenburg AB, a GU Ventures-based platform company at the University of Gothenburg. The authors report no conflicts of interest.

\section{References}

Ah Mew, N., Loewenstein, J.B., Kadom, N., Lichter-Konecki, U., Gropman, A.L., Martin, J.M., Vanderver, A., 2011. MRI features of 4 female patients with pyruvate dehydrogenase E1 alpha deficiency. Pediatr Neurol. 45(1), 57-59.

Cecchini, S., Regnicolo, L., Polonara, G., Sallei, M., Cesaroni, E., Tavoni, M.A., Zamponi, N., 2007. Leigh syndrome: an MR study of three cases. Neuroradiol J. 20(1), 48-55.

Darin, N., Oldfors, A., Moslemi, A.R., Holme, E., Tulinius, M., 2001. The incidence of mitochondrial encephalomyopathies in childhood: clinical features and morphological, biochemical, and DNA anbormalities. Ann. Neurol. 49(3), 377-383.

Debray, F.G., Mitchell, G.A., Allard, P., Robinson, B.H., Hanley, J.A., Lambert, M., 2007. Diagnostic accuracy of blood lactate-to-pyruvate molar ratio in the differential diagnosis of congenital lactic acidosis. Clin Chem. 53(5), 916-921.

Haas, R.H., Parikh, S., Falk, M.J., Saneto, R.P., Wolf, N.I., Darin, N., Wong, L.J., Cohen, B.H., Naviaux, R.K., Mitochondrial Medicine Society's Committee on Diagnosis, 2008. The in-depth evaluation of suspected mitochondrial disease. Mol. Genet. Metab. 94(1), 16-37.

Lin, M.T., Beal, M.F., 2006. Mitochondrial dysfunction and oxidative stress in neurodegenerative diseases. Nature 443(7113), 787-795.

Lu, C.H., Macdonald-Wallis, C., Gray, E., Pearce, N., Petzold, A., Norgren, N., Giovannoni, G., Fratta, P., Sidle, K., Fish, M., Orrell, R., Howard, R., Talbot, K., Greensmith, L., Kuhle, J., 
Turner, M.R., Malaspina, A., 2015. Neurofilament light chain: A prognostic biomarker in amyotrophic lateral sclerosis. Neurology. 84(22), 2247-2257.

Lunsing, R.J., Strating, K., de Koning, T.J., Sijens, P.E., 2017. Diagnostic value of MRSquantified brain tissue lactate level in identifying children with mitochondrial disorders. Eur Radiol. 27(3), 976-984.

Magner, M., Szentiványi, K., Svandová, I., Ješina, P., Tesařová, M., Honzík, T., Zeman, J., 2011. Elevated CSF-lactate is a reliable marker of mitochondrial disorders in children even after brief seizures. Eur J Paediatr Neurol. 15(2), 101-108.

Magnoni, S., Esparza, T.J., Conte, V., Carbonara, M., Carrabba, G., Holtzman, D.M., Zipfel, G.J., Stocchetti, N., Brody, D.L., 2012. Tau elevations in the brain extracellular space correlate with reduced amyloid- $\beta$ levels and predict adverse clinical outcomes after severe traumatic brain injury. Brain. 135(Pt 4), 1268-1280.

Mattsson, N., Andreasson, U., Zetterberg, H., Blennow, K.; Alzheimer's Disease Neuroimaging Initiative, 2017. Association of plasma Neurofilament Light with neurodegeneration in patients with Alzheimer disease. JAMA Neurol. 74(5), 557-566.

Moslemi, A.R., Darin, N., 2007. Molecular genetic and clinical aspects of mitochondrial disorders in childhood. Mitochondrion 7(4), 241-252.

Nylén, K., Csajbok, L.Z., Ost, M., Rashid, A., Karlsson, J.E., Blennow, K., Nellgård, B., Rosengren, L., 2006. CSF -neurofilament correlates with outcome after aneurysmal subarachnoid hemorrhage. Neurosci Lett. 404(1-2), 132-136.

Olsson, A., Vanderstichele, H., Andreasen, N., De Meyer, G., Wallin, A., Holmberg, B., Rosengren, L., Vanmechelen, E., Blennow, K., 2005. Simultaneous measurement of betaamyloid(1-42), total tau, and phosphorylated tau (Thr181) in cerebrospinal fluid by the xMAP technology. Clin Chem. 51(2), 336-345.

Olsson, B., Lautner, R., Andreasson, U., Öhrfelt, A., Portelius, E., Bjerke, M., Hölttä, M., Rosén, C., Olsson, C., Strobel, G., Wu, E., Dakin, K., Petzold, M., Blennow, K., Zetterberg, H., 2016. CSF and blood biomarkers for the diagnosis of Alzheimer's disease: a systematic review and meta-analysis. Lancet Neurol. 15(7), 673-684.

Reddy, P.H., 2009. Role of mitochondria in neurodegenerative diseases: mitochondria as a therapeutic target in Alzheimer's disease. CNS spectrums 14(8 Suppl 7), 8-13; discussion 1618.

Rosengren, L.E., Wikkelsø, C., Hagberg, L., 1994. A sensitive ELISA for glial fibrillary acidic protein: application in CSF of adults. J Neurosci Methods. 51(2), 197-204. 
Shahim, P., Gren, M., Liman, V., Andreasson, U., Norgren, N., Tegner, Y., Mattsson, N., Andreasen, N., Öst, M., Zetterberg, H., Nellgård, B., Blennow, K., 2016a. Serum neurofilament light protein predicts clinical outcome in traumatic brain injury. Sci Rep. 6, 36791.

Shahim, P., Tegner, Y., Gustafsson, B., Gren, M., Ärlig, J., Olsson, M., Lehto, N., Engström, Å., Höglund, K., Portelius, E., Zetterberg, H., Blennow, K., 2016b. Neurochemical aftermath of repetitive mild traumatic brain injury. JAMA Neurol. 73(11), 1308-1315.

Shahim, P., Månsson, J.E., Darin, N., Zetterberg, H., Mattsson, N., 2013a. Cerebrospinal fluid biomarkers in neurological diseases in children. Eur J Paediatr Neurol. 17(1), 7-13.

Shahim, P., Darin, N., Andreasson, U., Blennow, K., Jennions, E., Lundgren, J., Månsson, J.E., Naess, K., Törnhage, C.J., Zetterberg, H., Mattsson, N., 2013b. Cerebrospinal fluid brain injury biomarkers in children: a multicenter study. Pediatr Neurol. 49(1), 31-39.e2.

Skillbäck, T., Farahmand, B., Bartlett, J.W., Rosén, C., Mattsson, N., Nägga, K., Kilander, L., Religa, D., Wimo, A., Winblad, B., Rosengren, L., Schott, J.M., Blennow, K., Eriksdotter, M., Zetterberg, H., 2014. CSF neurofilament light differs in neurodegenerative diseases and predicts severity and survival. Neurology. 83(21), 1945-1953.

Serrano, M., García-Silva, M.T., Martin-Hernandez, E., O'Callaghan Mdel, M., Quijada, P., Martinez-Aragón, A., Ormazábal, A., Blázquez, A., Martín, M.A., Briones, P., LópezGallardo, E., Ruiz-Pesini, E., Montoya, J., Artuch, R., Pineda, M., 2010. Kearns-Sayre syndrome: cerebral folate deficiency, MRI findings and new cerebrospinal fluid biochemical features. Mitochondrion. 10(5), 429-432.

Sofou, K., De Coo, I.F., Isohanni, P., Ostergaard, E., Naess, K., De Meirleir, L., Tzoulis, C., Uusimaa, J., De Angst, I.B., Lönnqvist, T., Pihko, H., Mankinen, K., Bindoff, L.A., Tulinius, M., Darin, N., 2014. A multicenter study on Leigh syndrome: disease course and predictors of survival. Orphanet J Rare Dis. 9(52).

Sofou, K., Steneryd, K., Wiklund, L.M., Tulinius, M., Darin, N., 2013. MRI of the brain in childhood-onset mitochondrial disorders with central nervous system involvement. Mitochondrion 13(4), 364-371.

Tortorella, C., Direnzo, V., Taurisano, P., Romano, R., Ruggieri, M., Zoccolella, S., Mastrapasqua, M., Popolizio, T., Blasi, G., Bertolino, A., Trojano, M., 2015. Cerebrospinal fluid neurofilament tracks fMRI correlates of attention at the first attack of multiple sclerosis. Mult Scler. 21(4), 396-401. 
Tulinius, M.H., Holme, E., Kristiansson, B., Larsson, N.G., Oldfors, A., 1991a. Mitochondrial encephalomyopathies in childhood. I. Biochemical and morphologic investigations. J. Pediatr. 119(2), 242-250.

Tulinius, M.H., Holme, E., Kristiansson, B., Larsson, N.G., Oldfors, A., 1991b. Mitochondrial encephalomyopathies in childhood. II. Clinical manifestations and syndromes. J. Pediatr. 119(2), 251-259.

Zetterberg, H., Hietala, M.A., Jonsson, M., Andreasen, N., Styrud, E., Karlsson, I., Edman, A., Popa, C., Rasulzada, A., Wahlund, L.O., Mehta, P.D., Rosengren, L., Blennow, K., Wallin, A., 2006. Neurochemical aftermath of amateur boxing. Arch Neurol. 63(9), 1277-1280.

Zetterberg, H., Skillbäck, T., Mattsson, N., Trojanowski, J.Q., Portelius, E., Shaw, L.M., Weiner, M.W., Blennow, K.; Alzheimer's Disease Neuroimaging Initiative, 2016. Association of cerebrospinal fluid Neurofilament Light concentration with Alzheimer disease progression. JAMA Neurol. 73(1), 60-67. 


\section{Figure Legends}

Figure 1. Flow chart of enrolled patients.

Figure 2. Concentrations of blood lactate, CSF lactate and CSF NF-L between patients with mitochondrial encephalopathy $(n=46)$ and controls $(n=22)$

Patients with mitochondrial encephalopathy showed elevated CSF lactate and NF-L but unchanged blood lactate. Error bars indicate median and interquartile range.

$C S F$, cerebrospinal fluid; $N F-L$, neurofilament light.

Figure 3. Diagnostic utility of the biomarkers. AUROC for CSF lactate, NF-L and other biomarkers.

$A U R O C$, areas under the receiver-operating characteristics; CSF, cerebrospinal fluid; NF-L, neurofilament light; T-tau, total-tau; P-tau; phosphorylated tau; GFAp; glial fibrillary acidic protein; Ab42, amyloid- $\beta 42$.

Figure 4. Brain MRI of a 2.5 years old boy with Leigh syndrome and SLC19A3 mutation. Axial T2-weighted image (a) shows increased signal intensity in putamen, caudate nuclei and ventromedial thalamic nuclei bilaterally. Elevated lactate peak (thick arrow) is seen in magnetic resonance spectroscopy at the region of the basal ganglia $(b, c)$.

Figure 5. Estimated survival based on the Cox proportional hazard models.

(A) CSF NF-L, (B) CSF-lactate, (C) T-tau, (D) P-tau, (E) GFAp, and (F) Ab42. The models were adjusted for age, diagnosis and sex. The survival estimates are shown for quartiles of biomarker concentrations.

CSF, cerebrospinal fluid; NF-L, neurofilament light; T-tau, total-tau; P-tau; phosphorylated tau; GFAp; glial fibrillary acidic protein; Ab42, amyloid- $\beta 42$. 
Table 1

\begin{tabular}{|c|c|c|c|}
\hline Characteristics & $\begin{array}{l}\text { Controls } \\
(\mathrm{N}=\mathbf{2 2})\end{array}$ & $\begin{array}{l}\text { Mitochondrial } \\
\text { encephalopathy } \\
(\mathbf{N}=46)\end{array}$ & $P$ value \\
\hline Age at LP, years & $8.0(4-15)$ & $4.0(1.5-14)$ & 0.25 \\
\hline Sex, M:F (\% F) & $13: 9(41)$ & $20: 26(56)$ & 0.23 \\
\hline Blood lactate, $\mathrm{mmol} / \mathrm{L}$ & $1.1(0.7-2.1)$ & $1.5(0.7-2.4)$ & 0.21 \\
\hline CSF lactate, $\mathrm{mmol} / \mathrm{L}$ & $1.5(1.4-2.0)$ & $2.8(2.1-3.7)$ & $<0.001$ \\
\hline NF-L, ng/L & $210(127-379)$ & $2011(746-7194)$ & $<0.001$ \\
\hline T-tau, ng/L & $75(68-137)$ & $75(75-267)$ & 0.17 \\
\hline P-tau, ng/L & $42(29-56)$ & $40(27-57)$ & 0.86 \\
\hline GFAp, ng/L & $184(127-277)$ & $265(112-407)$ & 0.13 \\
\hline $\mathrm{A} \beta 42, \mathrm{ng} / \mathrm{L}$ & $611(455-763)$ & $497(411-649)$ & 0.20 \\
\hline \multicolumn{4}{|c|}{$\begin{array}{l}\text { LP, lumbar puncture; M, males; F, females; CSF; cerebrospinal fluid; T-tau, total- } \\
\text { tau; NF-L, neurofilament light; GFAp; glial fibrillary acidic protein; P-tau } \\
\text { phosphorylated tau; Ab42, amyloid- } \beta 42 \text {. Values are presented as median } \\
\text { (interquartile range). }\end{array}$} \\
\hline
\end{tabular}


Table 2

\begin{tabular}{|c|c|c|c|c|c|c|c|c|c|c|}
\hline Variables & $\begin{array}{l}\text { Alpers- } \\
\text { Huttenl } \\
\text { ocher } \\
\text { syndro } \\
\text { me } \\
\end{array}$ & PDC deficiency & KSS & Leigh syndrome & LE & MELAS & MERRF & Non-syndromic ME & $\begin{array}{l}\text { Mitochondri } \\
\text { al myopathy }\end{array}$ & Controls \\
\hline $\mathrm{N}$ & 1 & 6 & 5 & 16 & 1 & 9 & 4 & 4 & 6 & 16 \\
\hline Genetic findings & POLG1 & PDHA1, PDHX & $\begin{array}{l}\text { Mitochondrial } \\
\text { DNA deletion }\end{array}$ & $\begin{array}{l}\text { MT-ATP6, MT- } \\
N D 1, M T-N D 5, \\
M T-T W, S U R F 1, \\
\text { SLC19A3, } \\
\text { BCS1L, SUCLG1, } \\
\text { SUCLA2 }\end{array}$ & $\begin{array}{l}\text { NDU } \\
F V 1\end{array}$ & $M T-T L 1$ & $M T-T K$ & $\begin{array}{l}\text { MT-ATP6, POLG1, } \\
M T-T L 1\end{array}$ & $\begin{array}{l}\text { Mitochondrial } \\
\text { DNA } \\
\text { depletion, } \\
M T-T N, M T- \\
T L 1, I S C U\end{array}$ & $\mathrm{NA}$ \\
\hline $\begin{array}{l}\text { Lesions on brain } \\
\text { neuroimaging } \\
\text { (signal changes } \\
\text { and/or atrophy) }\end{array}$ & None & $\begin{array}{l}\mathrm{BG}, \mathrm{CB}, \mathrm{CC}, \\
\text { cortex, thalamus, } \\
\text { WM }\end{array}$ & $\begin{array}{l}\mathrm{BG}, \mathrm{CB}, \\
\text { midbrain, pons, } \\
\text { medulla, } \\
\text { thalamus, WM }\end{array}$ & $\begin{array}{l}\mathrm{BG}, \mathrm{CB}, \\
\text { midbrain, pons, } \\
\text { WM }\end{array}$ & $\begin{array}{l}\mathrm{CB}, \\
\mathrm{WM}\end{array}$ & $\mathrm{CB}$, cortex, WM & None & Cortex, thalamus & NA & $\mathrm{NA}$ \\
\hline $\begin{array}{l}\text { CSF-lactate, } \\
\mathrm{mmol} / \mathrm{L}\end{array}$ & $\begin{array}{l}1.4 \\
-\end{array}$ & $\begin{array}{l}3.1(2.6-4.8) \\
P=0.011\end{array}$ & $\begin{array}{l}3.2(1.8-4.0) \\
P=0.017\end{array}$ & $\begin{array}{l}2.9(1.2-5.7) \\
P=0.00017\end{array}$ & $\begin{array}{l}3.5 \\
-\end{array}$ & $\begin{array}{l}4.7(1.7-7.8) \\
P<0.0001\end{array}$ & $\begin{array}{l}1.9(1.4-2.5) \\
P=0.58\end{array}$ & $\begin{array}{l}2.5(2.1-2.8) \\
P=0.14\end{array}$ & $2.3(1.6-2.5)$ & $\begin{array}{l}1.5(1.2- \\
2.5)\end{array}$ \\
\hline NF-L, ng/L & $\begin{array}{l}3152 \\
-\end{array}$ & $\begin{array}{l}1812(127-18391) \\
P=0.038\end{array}$ & $\begin{array}{l}2117(313-5467) \\
P=0.53\end{array}$ & $\begin{array}{l}1662(210-29146) \\
P=0.011\end{array}$ & $\begin{array}{l}7058 \\
-\end{array}$ & $\begin{array}{l}4675(154-16347) \\
P=0.033\end{array}$ & $\begin{array}{l}818(500-1904) \\
P=0.83\end{array}$ & $\begin{array}{l}1277(887-14192) \\
P=0.30\end{array}$ & $\begin{array}{l}301(121- \\
1224)\end{array}$ & $\begin{array}{l}210(127- \\
379)\end{array}$ \\
\hline T-tau, ng/L & $\begin{array}{l}152 \\
-\end{array}$ & $\begin{array}{l}106(60-727) \\
P=0.56\end{array}$ & $\begin{array}{l}127(75-1443) \\
P=0.45\end{array}$ & $\begin{array}{l}90(5-12247) \\
P=0.077\end{array}$ & $\begin{array}{l}13 \\
-\end{array}$ & $\begin{array}{l}263(9-1404) \\
P=0.47\end{array}$ & $\begin{array}{l}75(26-75) \\
P=0.89\end{array}$ & $\begin{array}{l}106(28-158) \\
P=0.76\end{array}$ & $78(30-136)$ & $75(68-137)$ \\
\hline P-tau, ng/L & $\begin{array}{l}170 \\
-\end{array}$ & $\begin{array}{l}42(27-242) \\
P=0.99\end{array}$ & $\begin{array}{l}79(35-327) \\
P=0.035\end{array}$ & $\begin{array}{l}49(24-626) \\
P=0.50\end{array}$ & $\begin{array}{l}51 \\
-\end{array}$ & $\begin{array}{l}40(25-77) \\
P=0.49\end{array}$ & $\begin{array}{l}41(25-156) \\
P=0.75\end{array}$ & $\begin{array}{l}106(28-158) \\
P=0.45\end{array}$ & $78(30-136)$ & $42(29-56)$ \\
\hline GFAp, ng/L & $\begin{array}{l}408 \\
-\end{array}$ & $\begin{array}{l}225(112-672) \\
P=0.75\end{array}$ & $\begin{array}{l}407(244-1370) \\
P=0.80\end{array}$ & $\begin{array}{l}197(106-14282) \\
P=0.16\end{array}$ & $\begin{array}{l}271 \\
-\end{array}$ & $\begin{array}{l}389(112-7605) \\
P=0.43\end{array}$ & $\begin{array}{l}139(112-308) \\
P=0.80\end{array}$ & $\begin{array}{l}195(108-839) \\
P=0.97\end{array}$ & $178(112-352)$ & $\begin{array}{l}184(127- \\
277)\end{array}$ \\
\hline $\mathrm{A} \beta 42, \mathrm{ng} / \mathrm{L}$ & 971 & $\begin{array}{l}400(259-598) \\
P=0.019\end{array}$ & $\begin{array}{l}1239(501-884) \\
P=0.0006\end{array}$ & $\begin{array}{l}480(301-780) \\
P=0.080\end{array}$ & $\begin{array}{l}560 \\
- \\
\end{array}$ & $\begin{array}{l}486(370-884) \\
P=0.69\end{array}$ & $\begin{array}{l}535(366-765) \\
P=0.84\end{array}$ & $\begin{array}{l}548(391-1178) \\
P=0.35\end{array}$ & $562(377-673)$ & $\begin{array}{l}611(455- \\
763)\end{array}$ \\
\hline
\end{tabular}

NA, not available/applicable; PDC, pyruvate dehydrogenase complex; LE, leukoencephalopathy; MELAS, mitochondrial encephalomyopathy with lactic acidosis and stroke-like episodes; MERRF,

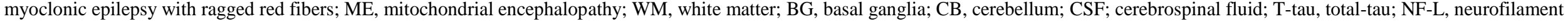
light; GFAp; glial fibrillary acidic protein; P-tau; phosphorylated tau; Ab42, amyloid- $\beta 42$.

Values are presented as median (range). $P$ values are from linear regression models co-varied for age at the time of lumbar puncture and sex, with the control group as reference.

Alpers-Huttenlocher syndrome and LE were excluded from the models due to limited number of patients. 